\title{
Exercise is an antigen for vaccination: first series of scientific evidence
}

\author{
Yong-Seok Jee (D) https://orcid.org/0000-0001-6797-0843
}

An antigen is a molecule that binds specifically to an antibody and can stimulate the production of antibodies. A vaccine is a biological preparation that provides immunity to a particular disease. A vaccine contains an agent that appears to be a disease-causing microorganism and is often made from weakened or destroyed pathogens. The agent causes the body's immune system to recognize it as a threat, destroys it, and then remembers it so that the immune system can more easily recognize and eradicate such agents in future encounters (Aldous and Dong, 2018). The administration of vaccines is known as vaccination, which has been widely studied and verified to be effective (Fiore et al., 2009).

Exercise provides an effect similar to a vaccine by inducing physiological stress to the human body. That is, when certain physical stimuli are applied to the human body, positive or negative changes are made to overcome the stimuli or to be defeated by it. The former can make the body healthier, while the latter can damage the body. Antigen to become a vaccine is also beneficial to health because it makes an antibody for human body when administered to the human body in an appropriate amount, but if it is overdosed, it may harm health rather than making antibody. In this regard, exercise can be called an antigen for the vaccine.

Vaccination is a procedure in which antibodies or cell mediated responses are induced by exposing the immune system to an antigen. The factors that lead to changes in the vaccination response is very important to understand in terms of clinical and public health (Edwards and Booy, 2013). The role of exercise as a form of vaccination has both acute and chronic benefits. In 1996, World
Health Organization reported that acute physiological benefits of exercise include improved sleep, controlled blood glucose levels, and increased catecholamine activity and that chronic effects of continuous exercise include improved cardiovascular performance, increased strength and muscle endurance, improved flexibility, reduced fat mass, and improved lipid composition. However, as mentioned above, if the exercise is overdosed, it causes a lot of negative results from muscle damage to cell destruction.

The most effective method of preventing infectious diseases is vaccination (Bustreo et al., 2015). Vaccination has provided widespread immunity to various diseases. In 2017, Feldstein et al. (2017) report that the improvements in national immunization program performance are necessary to reach and sustain high vaccination coverage to increase protection from vaccine-preventable diseases for all persons. In the future, exercise may be included in the list of licensed vaccines for preventing disease and so on (Pascoe et al., 2014). Vaccination programs, although credited for reducing infectious diseases, are limited by vaccine efficacy. This can be problematic in populations with low immune function. However, since exercise has been identified as a behavioral factor that can improve immune function, it can serve as a form of vaccination against certain diseases or infections.

\section{CONFLICT OF INTEREST}

No potential conflict of interest relevant to this article was reported. 


\section{REFERENCES}

Aldous AR, Dong JZ. Personalized neoantigen vaccines: a new approach to cancer immunotherapy. Bioorg Med Chem 2018;26:2842-2849.

Bustreo F, Okwo-Bele JM, Kamara L. World Health Organization perspectives on the contribution of the Global Alliance for Vaccines and Immunization on reducing child mortality. Arch Dis Child 2015;100 Suppl 1:S34-37.

Edwards KM, Booy R. Effects of exercise on vaccine-induced immune responses. Hum Vaccin Immunother 2013;9:907-910.

Feldstein LR, Mariat S, Gacic-Dobo M, Diallo MS, Conklin LM, Wallace AS. Global routine vaccination coverage, 2016. MMWR Morb Mortal Wkly Rep 2017;66:1252-1255.
Fiore AE, Bridges CB, Cox NJ. Seasonal influenza vaccines. Curr Top Microbiol Immunol 2009;333:43-82.

Pascoe AR, Fiatarone Singh MA, Edwards KM. The effects of exercise on vaccination responses: a review of chronic and acute exercise interventions in humans. Brain Behav Immun 2014;39:33-41.

World Health Organization. Regional Office for Europe. The Heidelberg guidelines for promoting physical activity among older persons. Copenhagen: WHO Regional Office for Europe; 1996.

Research Institute of Sports and Industry Science, Hanseo University, 46 Hanseo 1-ro, Haemi-myeon, Seosan 31962, Korea E-mail: jeeys@hanseo.ac.kr 